\title{
Effectiveness of malaria control interventions in Madagascar: a nationwide case-control survey
}

Thomas Kesteman ${ }^{1,2,3^{*}}$ D, Milijaona Randrianarivelojosia ${ }^{1}$, Vaomalala Raharimanga ${ }^{4}$, Laurence Randrianasolo ${ }^{4}$, Patrice Piola ${ }^{4}$ and Christophe Rogier ${ }^{1,2,5}$

\begin{abstract}
Background: Madagascar, as other malaria endemic countries, depends mainly on international funding for the implementation of malaria control interventions (MCl). As these funds no longer increase, policy makers need to know whether these $\mathrm{MCl}$ actually provide the expected protection. This study aimed at measuring the effectiveness of MCl deployed in all transmission patterns of Madagascar in 2012-2013 against the occurrence of clinical malaria cases.
\end{abstract}

Methods: From September 2012 to August 2013, patients consulting for non-complicated malaria in 31 sentinel health centres (SHC) were asked to answer a short questionnaire about long-lasting insecticidal nets (LLIN) use, indoor residual spraying (IRS) in the household and intermittent preventive treatment of pregnant women (IPTp) intake. Controls were healthy all-ages individuals sampled from a concurrent cross-sectional survey conducted in areas surrounding the SHC. Cases and controls were retained in the database if they were resident of the same communes. The association between Plasmodium infection and exposure to $\mathrm{MCI}$ was calculated by multivariate multilevel models, and the protective effectiveness (PE) of an intervention was defined as 1 minus the odds ratio of this association.

Results: Data about 841 cases (out of 6760 cases observed in SHC) and 8284 controls was collected. The regular use of LLIN provided a significant $51 \%$ PE (95\% Cl [16-71]) in multivariate analysis, excluding in one transmission pattern where PE was $-11 \%$ (95\% Cl [-251 to 65]) in univariate analysis. The PE of IRS was $51 \%$ (95\% Cl [31-65]), and the PE of exposure to both regular use of LLIN and IRS was $72 \%$ (95\% CI [28-89]) in multivariate analyses. Vector control interventions avoided yearly over 100,000 clinical cases of malaria in Madagascar. The maternal PE of IPTp was $73 \%$.

Conclusions: In Madagascar, LLIN and IRS had good PE against clinical malaria. These results may apply to other countries with similar transmission profiles, but such case-control surveys could be recommended to identify local failures in the effectiveness of $\mathrm{MCl}$.

Keywords: Malaria, Morbidity, Prevention and control, Case-control studies, Health surveys, Vector control, Insecticide-treated bed nets

\section{Background}

Malaria control has been intensified in the last decade, leading to an important reduction in its incidence [1]. In Madagascar, the incidence of clinical malaria in

\footnotetext{
${ }^{*}$ Correspondence: thomask@pasteur.mg

${ }^{1}$ Malaria Research Unit, Institut Pasteur de Madagascar, BP 1274,

101 Avaradoha, Antananarivo, Madagascar

Full list of author information is available at the end of the article
}

outpatient wards declined by $81 \%$, in inpatient wards by $69 \%$ and mortality by $75 \%$ between 2000 and 2010 [2]. This reduction in malaria burden could be attributed to the intensification and scale up of malaria control interventions (MCI), but it remains unknown what part of this decline was attributable to the MCIs. It is also not known which intervention(s) within the package is(are) most effective [3]. Moreover, other factors may have played a role that would balance the optimism 
arising from this success, e.g. increase of urbanization [4], climate change [5], and changes in socio-economic factors [6]. Roll-out of rapid diagnostic tests (RDT) may cause a false impression of decline in incidence because false positive cases-formerly diagnosed on the sole base of symptoms-are excluded from cases count [7]. Now that international funding for malaria control stopped increasing, it becomes of public health and strategic importance to measure the actual effectiveness of MCIs, in order to fund what is really working. In this context, the present study was aimed to evaluate whether interventions actually deployed performed well by measuring their individual effectiveness, their coverage and, by multiplication of these two values, their community effectiveness $[8,9]$. Although the level of evidence generated by observational studies don't reach the one generated by randomized controlled trials (RCTs) it would be unethical to conduct RCTs-and thus intentionally leave individuals unprotected-for policy guidance.

The study was named MEDALI-a French acronym standing for Mission d'Etude des Déterminants de l'Accès aux Méthodes de Lutte antipaludique et de leur Impactand took place in Madagascar in 2012-2013. The design and methodology of the overall MEDALI project has been previously described [10]. The primary objective of the study presented here was the evaluation of the effectiveness against incidence of non-complicated clinical malaria of long-lasting insecticidal nets (LLIN) and indoor residual spraying (IRS) in the overall population. The secondary objective was the evaluation of the effectiveness against incidence of non-complicated clinical malaria of intermittent preventive treatment in pregnancy (IPTp) in a population of pregnant women or women having recently delivered.

\section{Methods}

\section{Study design}

Districts of Madagascar are divided into five main operational zones (Fig. 1), which correspond to the transmission patterns of Madagascar [11]. The two coastal regions exhibit hyperendemic patterns with a transmission lasting all year in the East and more than 6 months per year in the West. In the central highlands, the transmission is unstable, and episodic or epidemic. In the fringe areas, i.e. at intermediate altitudes, the transmission pattern is seasonal, lasting from November to May (rainy season). In the South, the period of transmission is short and episodic. Fringe, central highlands and South are prone to outbreaks.

The selection of study sites was based on a network of sentinel health centres (SHC) for surveillance of fever-associated diseases that has been established in order to cover all the ecosystems of Madagascar [12].
Each location where at least one SHC existed in 2012 was included in the study, thus defining 31 study sites. All malaria transmission patterns were represented: 13 sites were located in the Western transmission pattern, seven in the East, five in the Fringe, four in the Central Highlands and two in the South (Fig. 1). These patterns encompass respectively 21.0, 27.5, 13.7, 31.9 and $5.9 \%$ of Malagasy population. The design consisted in recruiting non-complicated clinical malaria cases in health facilities belonging to the SHC network, and controls in the population living in their catching same areas.

\section{Inclusion of cases}

All 31 SHCs were proposed to participate in the study protocol. In the participating SHCs, patients presenting with clinical malaria cases or their tutors were asked to answer a short one-page questionnaire about sociodemographic data and exposure to MCIs: LLIN, IRS and IPTp. Inclusion criteria were (1) fever, i.e., axillary temperature $\geq 37.5{ }^{\circ} \mathrm{C}$ [13] or self-reported symptoms of fever; (2) RDT or microscopy positive for any Plasmodium species; (3) age $\geq 6$ months; and (4) informed consent of the patient or his/her tutor. CareStart ${ }^{\circledR}$ Malaria RDT (Access Bio Inc., Monmouth Junction (NJ), USA) was used, which is the RDT commonly used in the public health system in Madagascar. Cases were retained in the database if they came from the same commune as controls. Data were collected from September 2012 to August 2013.

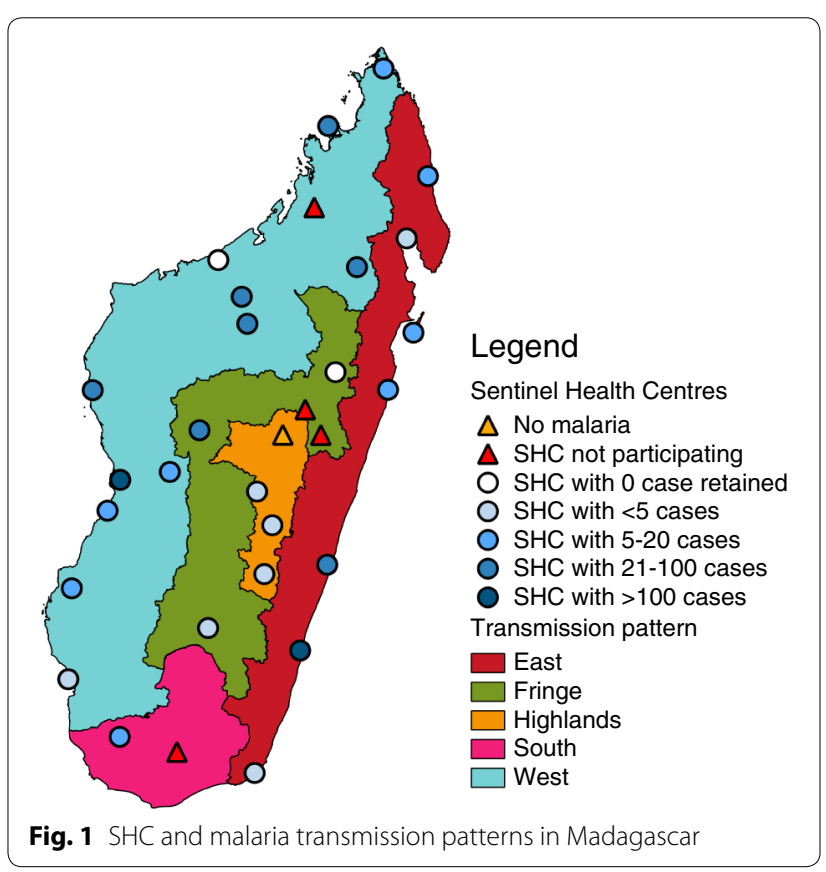




\section{Inclusion of controls}

Controls were selected from a cross-sectional survey (CSS), which took place in the same areas in the context of the very same MEDALI project, between September 2012 and January 2013. The methodology of this CSS has been previously described [10]. Briefly, it was a crosssectional household survey in which a two-stage cluster sampling technique was used to randomly select two fokontanys (smallest administrative subdivision in Madagascar) near each SHC. In each fokontany, the investigators followed a random path to include 50 households, i.e. approximately 225 individuals per site. The sample size of controls was calculated for a concomitant crosssectional survey, leaving controls in excess [10, 14, 15]. Heads of households and all members of the household eligible for the survey were interviewed about sociodemographic features and exposure to MCIs, their axillary temperature was measured, and a RDT (CareStart ${ }^{\circledR}$ Malaria) was performed. Inclusion criteria were: (1) age $\geq 6$ months, (2) having signed individual informed consent including agreement for blood sampling, and (3) being able to take a per os treatment in case of positive RDT. Parents or tutors signed and answered the questionnaire for minors and impaired individuals. Controls were retained in the database if they came from the same commune as cases, if they were permanent residents of the household, and if they had no malaria at the time of survey (i.e. fever or history of fever, and RDT or microscopy positive for any Plasmodium species), or in the last 3 months (i.e. diagnosis of malaria, or history of fever treated with anti-malarial drugs, or history of fever with unknown management).

\section{Sample size calculation}

The primary objective for sample size calculation was to detect the association between occurrence of clinical malaria due to any Plasmodium species, and exposure to MCI. It was assumed that at least three controls would be found for each case. A sample size of 800 cases and 2400 controls has a power of 87,70 , and $49 \%$ for detecting OR of $0.7,0.75$ and 0.8 , respectively, considering the following parameters: coverage of intervention of $50 \%$ in controls, cluster effect of 2 , and alpha risk of $5 \%$ [16].

\section{Data management and statistical analyses}

Bed net use was defined as "use every night during last 3 months" because it is more stringent than the "last night" definition $[10,14]$. The association between exposure to MCIs and being a case was estimated by generalized estimating equations models (GEE) taking into account an exchangeable within-site correlation structure using gee function on R software [17]. GEE models allow for a robust estimation of ORs and their confidence intervals while controlling for clustering [9]. Controls were neither matched with cases nor limited to three controls per case, but adjustment variables (age, sex, and transmission pattern) were forced in all models. All multivariate model fits were evaluated using binned residual plots $[18,19]$. Whether malaria transmission pattern, age less than 5 years, or season of detection of cases influenced the effectiveness of MCI was tested by introducing interaction terms in the models. Whenever season modified the effectiveness measured, the analysis was rerun on the cases that occurred in the same quarter as the collection of data on controls. The protective effectiveness (PE) of an intervention was defined as 1 minus the odds ratio of the exposure to this intervention as suggested previously [8].

\section{Estimation of the number of clinical cases of malaria avoided}

In order to evaluate the number of clinical cases of malaria prevented by each MCI with significant PE, the PE value was first multiplied by the coverage of the $\mathrm{MCI}$ in the general population, thus giving the proportion of cases avoided or "community effectiveness" (CE), as described previously [8]. The estimated number of cases avoided was defined as the annual number of clinical cases multiplied by $\mathrm{CE} /(1-\mathrm{CE})$. Coverage values were extracted from the concomitant CSS mentioned previously [10], and number of malaria cases in 2011 by districts was provided by the National Malaria Control Programme.

\section{Ethical considerations}

All surveys followed ethical principles according to the Helsinki Declaration. Informed consent was obtained from the individuals, or the parents/tutors of the children before inclusion. The protocol was approved by the National Ethic Committee of the Ministry of Public Health of Madagascar (approval \#CNE 57/MSANP/CE of July 24th, 2012).

\section{Results}

From September 2012 to August 2013, 6760 clinical malaria cases were observed in 30 SHCs. Four SHC did not participate and one had no malaria cases during the period (Figs. 1, 2). Among the remaining 6413 cases, 1582 questionnaires $(24.7 \%)$ were filled. The proportion of refusals among missing questionnaires is unknown. Individuals coming from the same communes as individuals included in the CSS accounted for $58.3 \%$ of the questionnaires sent. Among those, 841 questionnaires (91.1\%) were correctly filled, from 24 SHC (Fig. 2). Overall, 34 clusters (communes) were identified in all transmission patterns: $61.4 \%$ of cases occurred in the West, 


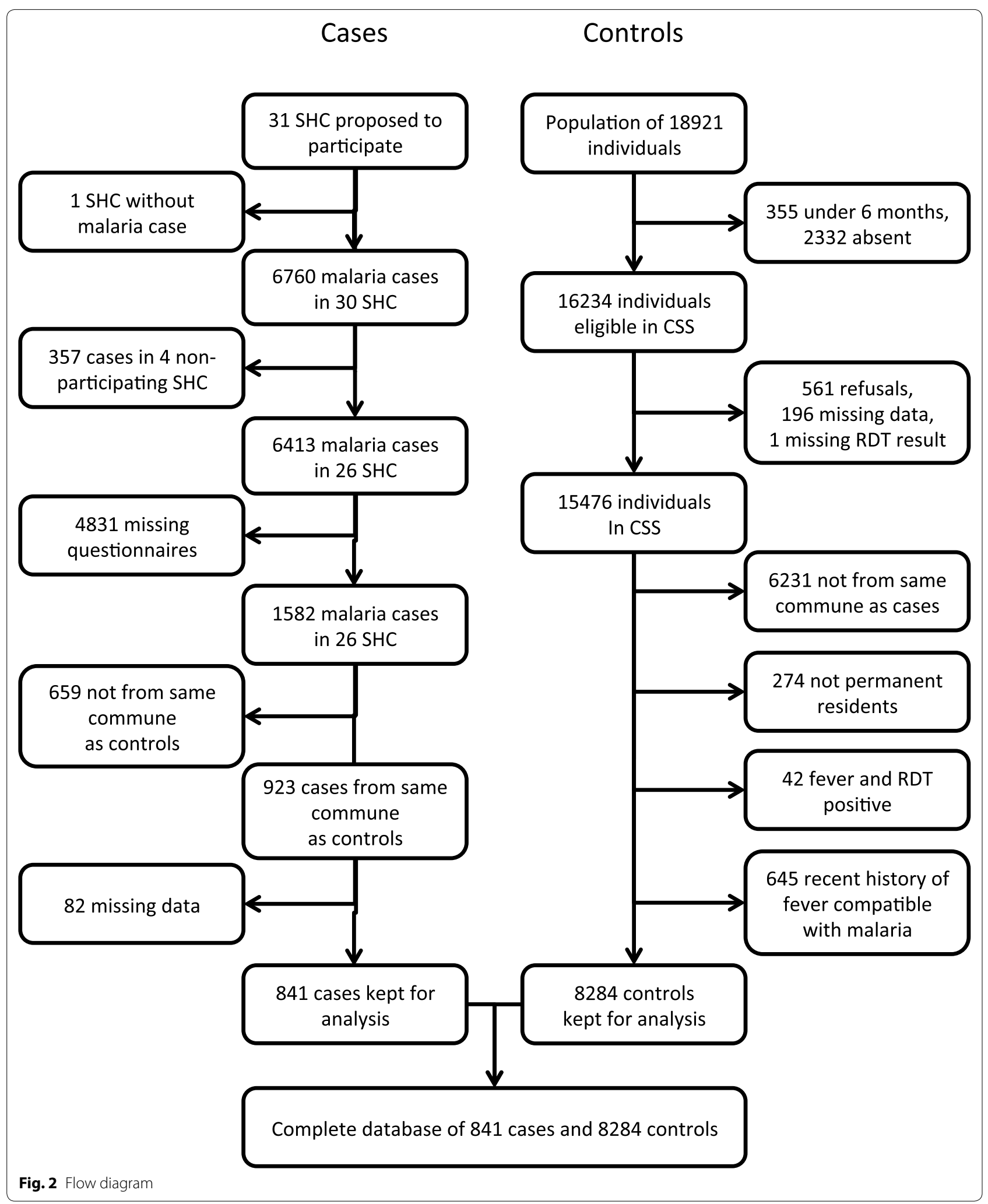


$30.0 \%$ in the East, $6.9 \%$ in the Fringe, $1.0 \%$ in the South, and $0.8 \%$ in the Highlands. The mean number of clinical malaria cases per cluster was 24.7 individuals (range 1-179). These episodes occurred between the end of September 2012 and mi-June 2013: $39.3 \%$ in spring, i.e. before December 31st, 2012; $47.8 \%$ in summer, i.e. between January 1st and March 31st, 2013; and $12.9 \%$ in fall, i.e. after April 1st, 2013.

In a population of 18,921 individuals, 15,476 were included in the CSS $[10,14]$. Among those, 6231 (40.3\%) were not comparable to cases since they were located in other communes, and 274 (1.8\%) were not permanent resident of the households where they had been identified. Of the remaining 8971 individuals, $42(0.5 \%)$ had an ongoing malaria episode and $645(7.2 \%)$ described a history of fever within the last 3 months that was compatible with an episode of malaria (Fig. 2). The study ended up with a database of 841 cases and 8284 controls, dispatched in 34 clusters (communes). The majority of the sample was from the Western transmission pattern (61.4\% of cases, $52.2 \%$ of controls), then came the East (30.0\% of cases and $26.5 \%$ of controls), and Fringe (6.9\% of cases and $8.1 \%$ of controls); the Highlands and the Southern transmission patterns encompassed seven and eight cases only (representing 0.8 and $1.0 \%$ of cases, respectively) and their controls represented 8.1 and $5.1 \%$ of controls, respectively.

\section{Bed nets}

In the areas where LLIN are distributed, i.e. Eastern, Western, Southern, and Fringe transmission patterns, the sample encompassed 31 clusters including 834 cases and 7617 controls. The use of LLIN every night was higher in controls (53.2\%) than in cases (39.3\%), and provided a significant $53 \%$ PE (95 \% CI [20-73]) in bivariate and $50 \%$ (95\% CI [16-70]) in multivariate analyses (Additional file 1). No significant interaction term was found between the season or the age under than 5 years old, and LLIN use. LLIN use among cases decreased from $43.3 \%$ in spring, to $37.4 \%$ in summer, and to $34.4 \%$ in fall, but this difference was not significant (Fisher's exact test, $\mathrm{p}>0.1$ ). A significant interaction term between the southern transmission pattern and LLIN use was identified ( $\mathrm{p}<0.001$ ), and separate analyses were conducted for the South and the rest of target zones. In the South, the association between LLIN use and clinical malaria was non- significant (OR 1.11, 95 \% CI [0.35-3.51]) in bivariate analysis. The small number of cases $(n=8)$ in the South precluded the possibility to conduct a multivariate analysis. In all other areas targeted by LLIN distribution campaigns, the use of LLIN every night provided a significant $51 \%$ PE (95 \% CI [16-71], Table 1), and the use of non-impregnated bed net (NIBN) a significant $37 \%$ PE in multivariate analysis (95\% CI [6-58], Table 1).
IRS

IRS campaigns took place in the Fringe, in most of Highlands, and in certain parts of Western and Southern transmission patterns. In these areas, living in a household that had been sprayed within the last 12 months provided a significant $54 \% \mathrm{PE}$ in bivariate and multivariate analyses (95\% CI [30-70] and [36-68], respectively). Models were run on a database of 11 clusters, including 199 cases and 2680 controls. No significant interaction term between the age under than 5 years old, or transmission pattern, and exposure to IRS was found. IRS coverage in the previous year among cases significantly decreased from $72.5 \%$ in the spring, to $43.5 \%$ in the summer, and to $4.8 \%$ in the fall (Fisher's exact test, $\mathrm{p}<0.001$ ). Interaction terms between season and IRS were also significant $(\mathrm{p}<0.001)$, and the final models were thus restricted to cases having occurred in the spring of 2012. In this analysis, 179 cases and 1125 controls belonging to five clusters in the Fringe, Western and Southern transmission patterns were analyzed. The PE of IRS was $50 \%$ in bivariate analysis (95\% CI [24-67]) and $51 \%$ in multivariate analysis (95\% CI [31-65], Table 2).

\section{Concurrent exposure to IRS and LLIN}

Given the above reported interactions, the analysis was restricted to cases occurring in the spring of 2012, and excluded the Southern transmission pattern, resulting in a sub-sample comprising four clusters, 173 cases and 916 controls in the Fringe and Western transmission patterns. In these clusters where IRS campaigns and LLIN distributions occurred, being exposed to both MCI provided a significant $72 \% \mathrm{PE}$ in bivariate and multivariate analyses (95 \% CI [22-90] and [28-89], respectively, Table 3). There was some association between exposure to both intervention and exposure to a single intervention, but it did not reach the significance threshold (OR against LLIN alone $0.75,95 \%$ CI [0.54-1.04], $\mathrm{p}=0.082$, and OR against IRS alone 0.63, [0.38-1.06], $\mathrm{p}=0.082$ ).

\section{IPTp}

In Madagascar, IPTp is proposed in all transmission patterns except in the Highlands. In these areas, having taken at least one dose of IPTp during pregnancy provided a $78 \% \mathrm{PE}$ in bivariate analysis and a $73 \% \mathrm{PE}$ in multivariate analysis, but these results were not statistically significant (Table 4).

\section{Estimation in the number of clinical malaria cases avoided by LLIN and IRS at the country level}

Considering number of malaria cases provided by National Malaria Control Programme for 2011 and coverage from the CSS mentioned previously [10], these interventions could have saved over 100,000 clinical 
Table $1 \mathrm{Bi}$ - and multivariate analyses of risk factors for developing a clinical malaria episode, including bed net use, in multivariate analyses, in zones targeted for LLIN distribution excluding the Southern transmission pattern (826 cases, 7192 controls, 29 communes)

\begin{tabular}{|c|c|c|c|c|c|c|c|}
\hline \multirow[t]{2}{*}{ Variable } & \multirow[t]{2}{*}{ Category } & \multirow[t]{2}{*}{$\mathrm{N}$ cases $(\%)$} & \multirow[t]{2}{*}{$\mathrm{N}$ controls (\%) } & \multicolumn{2}{|l|}{ Bivariate } & \multicolumn{2}{|l|}{ Multivariate } \\
\hline & & & & Crude OR [95\%Cl] & $\mathbf{p}$ & Adjusted OR $[95 \% \mathrm{Cl}]$ & $\mathbf{p}$ \\
\hline \multirow[t]{3}{*}{ Every night bed net use } & LLIN & $326(39.5)$ & $3959(55.0)$ & $0.47[0.27-0.80]$ & 0.005 & $0.49[0.29-0.84]$ & 0.009 \\
\hline & NIBN & $84(10.2)$ & $857(11.9)$ & $0.62[0.40-0.95]$ & 0.029 & $0.63[0.42-0.94]$ & 0.023 \\
\hline & None & $416(50.4)$ & $2376(33.0)$ & 1.00 & & 1.00 & \\
\hline \multirow[t]{7}{*}{ Age group } & $0-1$ year & $56(6.8)$ & $302(4.2)$ & $3.42[1.53-7.64]$ & 0.003 & $3.29[1.45-7.48]$ & 0.004 \\
\hline & $2-4$ years & $113(13.7)$ & $771(10.7)$ & $2.93[1.66-5.17]$ & $<0.001$ & $2.72[1.51-4.90]$ & $<0.001$ \\
\hline & $5-9$ years & $175(21.2)$ & $1228(17.1)$ & $2.70[1.79-4.06]$ & $<0.001$ & $2.44[1.63-3.65]$ & $<0.001$ \\
\hline & $10-15$ years & $169(20.5)$ & $1051(14.6)$ & $2.97[2.12-4.14]$ & $<0.001$ & $2.57[1.85-3.58]$ & $<0.001$ \\
\hline & $15-19$ years & $122(14.8)$ & $762(10.6)$ & $3.01[1.93-4.69]$ & $<0.001$ & $2.49[1.66-3.75]$ & $<0.001$ \\
\hline & 20-39 years & $137(16.6)$ & $1784(24.8)$ & $1.67[0.98-2.87]$ & 0.061 & $1.57[0.93-2.65]$ & 0.088 \\
\hline & $\geq 40$ years & $54(6.5)$ & $1294(18.0)$ & 1.00 & & 1.00 & \\
\hline \multirow[t]{2}{*}{ Sex } & Male & $447(54.1)$ & $3114(43.3)$ & 1.00 & & 1.00 & \\
\hline & Female & $379(45.9)$ & $4078(56.7)$ & $0.64[0.53-0.78]$ & $<0.001$ & $0.74[0.62-0.87]$ & $<0.001$ \\
\hline \multirow[t]{3}{*}{ Transmission pattern } & East & $252(30.5)$ & $2194(30.5)$ & $1.10[0.16-7.55]$ & 0.923 & $1.90[0.24-15.13]$ & 0.542 \\
\hline & Fringe & $58(7.0)$ & $670(9.3)$ & 1.00 & & 1.00 & \\
\hline & West & $516(62.5)$ & $4328(60.2)$ & $1.41[0.27-7.44]$ & 0.686 & $1.46[0.22-9.60]$ & 0.696 \\
\hline
\end{tabular}

Table $2 \mathrm{Bi}$ - and multivariate analyses of risk factors for developing a clinical malaria episode, including IRS the previous year in the household (179 cases, 1125 controls, 5 communes)

\begin{tabular}{|c|c|c|c|c|c|c|c|}
\hline \multirow[t]{2}{*}{ Variable } & \multirow[t]{2}{*}{ Category } & \multirow[t]{2}{*}{$\mathrm{N}$ cases $(\%)$} & \multirow[t]{2}{*}{$\mathrm{N}$ controls $(\%)$} & \multicolumn{2}{|l|}{ Bivariate } & \multicolumn{2}{|l|}{ Multivariate } \\
\hline & & & & Crude OR [95 \%Cl] & $\mathbf{p}$ & Adjusted OR [95 \%Cl] & $\mathbf{p}$ \\
\hline \multirow[t]{2}{*}{ IRS the previous year } & No & $92(51.4)$ & $778(69.2)$ & $0.50[0.33-0.76]$ & 0.001 & $0.49[0.35-0.69]$ & $<0.001$ \\
\hline & Yes & 87 (48.6) & $347(30.8)$ & 1.00 & & 1.00 & \\
\hline \multirow[t]{7}{*}{ Age group } & $0-1$ year & $19(10.6)$ & $37(3.3)$ & 26.08 [12.00-56.71] & $<0.001$ & $20.76[9.44-45.66]$ & $<0.001$ \\
\hline & $2-4$ years & $34(19.0)$ & $130(11.6)$ & $14.24[6.12-33.16]$ & $<0.001$ & $12.85[5.77-28.63]$ & $<0.001$ \\
\hline & $5-9$ years & $38(21.2)$ & $198(17.6)$ & $11.17[5.39-23.16]$ & $<0.001$ & $9.69[5.07-18.52]$ & $<0.001$ \\
\hline & $10-15$ years & $26(14.5)$ & $180(16.0)$ & $7.33[2.08-25.90]$ & 0.002 & $6.44[2.03-20.41]$ & 0.002 \\
\hline & $15-19$ years & $26(14.5)$ & $125(11.1)$ & $11.29[3.57-35.67]$ & $<0.001$ & $10.03[3.93-25.58]$ & $<0.001$ \\
\hline & 20-39 years & $33(18.4)$ & $247(22.0)$ & $7.34[2.77-19.43]$ & $<0.001$ & $6.45[2.81-14.81]$ & $<0.001$ \\
\hline & $\geq 40$ years & $3(1.7)$ & $208(18.5)$ & 1.00 & & 1.00 & \\
\hline \multirow[t]{2}{*}{ Sex } & Male & $98(54.7)$ & $476(42.3)$ & 1.00 & & 1.00 & \\
\hline & Female & $81(45.3)$ & $649(57.7)$ & $0.59[0.52-0.66]$ & $<0.001$ & $0.65[0.55-0.78]$ & $<0.001$ \\
\hline \multirow[t]{3}{*}{ Transmission pattern } & Fringe & $55(30.7)$ & $219(19.5)$ & 1.00 & & 1.00 & \\
\hline & West & $118(65.9)$ & $697(62.0)$ & $0.61[0.25-1.47]$ & 0.269 & $1.50[0.71-3.14]$ & 0.288 \\
\hline & South & $6(3.4)$ & $209(18.6)$ & $0.11[0.11-0.11]$ & $<0.001$ & $0.13[0.12-0.14]$ & $<0.001$ \\
\hline
\end{tabular}

malaria cases in one year (Table 5 ), $88 \%$ by LLIN and $12 \%$ by IRS.

\section{Discussion}

During the last decade, Madagascar has started and scaled up various interventions and policy changes regarding malaria control, resulting in dramatic decline in malaria burden, but the impact of each MCI cannot be disentangled by ecological studies [3]. This case-control study provided reliable information for guidance in policy making. In this study, vector control interventions demonstrated important and significant PE: LLIN provided a $51 \%$ PE (95 \% CI 16-71\%) and IRS a $51 \%$ PE (95\% CI 31-65) when controlling for major confounding variables. 
Table $3 \mathrm{Bi}$ - and multivariate analyses of risk factors for developing a clinical malaria episode, including bed net use and IRS (134 cases, 749 controls, 4 communes)

\begin{tabular}{|c|c|c|c|c|c|c|c|}
\hline \multirow[t]{2}{*}{ Variable } & \multirow[t]{2}{*}{ Category } & \multirow[t]{2}{*}{$\mathrm{N}$ cases $(\%)$} & \multirow[t]{2}{*}{ N controls $(\%)$} & \multicolumn{2}{|l|}{ Bivariate } & \multicolumn{2}{|l|}{ Multivariate } \\
\hline & & & & Crude OR [95 \%Cl] & $\mathbf{p}$ & Adjusted OR [95 \%Cl] & $\mathbf{p}$ \\
\hline \multirow{5}{*}{$\begin{array}{l}\text { Every night bed net use } \\
\text { and/or IRS the previous } \\
\text { year }\end{array}$} & LLIN use and IRS & $28(16.2)$ & $335(36.6)$ & $0.28[0.10-0.78]$ & 0.015 & $0.28[0.11-0.72]$ & 0.008 \\
\hline & No LLIN use and IRS & $60(34.7)$ & $317(34.6)$ & $0.43[0.25-0.74]$ & 0.003 & $0.44[0.26-0.74]$ & 0.002 \\
\hline & LLIN use and no IRS & $12(6.9)$ & $97(10.6)$ & $0.34[0.10-1.11]$ & 0.073 & $0.37[0.11-1.25]$ & 0.110 \\
\hline & NIBN use and no IRS & $6(3.5)$ & $18(2.0)$ & $1.00[0.78-1.30]$ & 0.972 & $0.90[0.75-1.09]$ & 0.287 \\
\hline & No bed net use and no IRS & $67(38.7)$ & $149(16.3)$ & 1.00 & & 1.00 & \\
\hline \multirow[t]{6}{*}{ Age group } & $0-1$ year & $19(11.0)$ & $31(3.4)$ & $5.69[3.35-9.69]$ & $<0.001$ & 4.78 [2.56-8.92] & $<0.001$ \\
\hline & $2-4$ years & $33(19.1)$ & $96(10.5)$ & $3.12[2.33-4.18]$ & $<0.001$ & $3.02[1.96-4.65]$ & $<0.001$ \\
\hline & $5-9$ years & $38(22.0)$ & $152(16.6)$ & 2.49 [1.95-3.19] & $<0.001$ & $2.38[1.81-3.12]$ & $<0.001$ \\
\hline & 10-15 years & $23(13.3)$ & $148(16.2)$ & 1.36 [0.83-2.23] & 0.218 & $1.27[0.70-2.31]$ & 0.440 \\
\hline & 15-19 years & $24(13.9)$ & $111(12.1)$ & 2.17 [1.52-3.09] & $<0.001$ & $1.97[1.48-2.62]$ & $<0.001$ \\
\hline & $\geq 20$ years & $36(20.8)$ & $378(41.3)$ & 1.00 & & 1.00 & \\
\hline \multirow[t]{2}{*}{ Sex } & Male & $94(54.3)$ & $386(42.1)$ & 1.00 & & 1.00 & \\
\hline & Female & 79 (45.7) & $530(57.9)$ & $0.59[0.54-0.66]$ & $<0.001$ & 0.68 [0.56-0.83] & $<0.001$ \\
\hline \multirow[t]{2}{*}{ Transmission pattern } & Fringe & $55(31.8)$ & $219(23.9)$ & 1.00 & & 1.00 & \\
\hline & West & $118(68.2)$ & $697(76.1)$ & $0.61[0.25-1.47]$ & 0.268 & 1.14 [0.94-1.38] & 0.196 \\
\hline
\end{tabular}

Table 4 Bi- and multivariate analyses of risk factors for developing a clinical malaria episode, including IPTp

\begin{tabular}{|c|c|c|c|c|c|c|c|}
\hline \multirow[t]{2}{*}{ Variable } & \multirow[t]{2}{*}{ Category } & \multirow[t]{2}{*}{$\mathrm{N}$ cases $(\%)$} & \multirow[t]{2}{*}{$\mathrm{N}$ controls (\%) } & \multicolumn{2}{|l|}{ Bivariate } & \multicolumn{2}{|l|}{ Multivariate } \\
\hline & & & & Crude OR [95 \%Cl] & $p$ & Adjusted OR $[95 \% \mathrm{Cl}]$ & $\mathrm{p}$ \\
\hline \multirow[t]{2}{*}{$\geq 1$ dose IPTp } & Yes & $3(25.0)$ & $11(57.9)$ & $0.22[0.04-1.29]$ & 0.093 & $0.27[0.04-1.89]$ & 0.187 \\
\hline & No & $9(75.0)$ & $8(42.1)$ & 1.00 & & 1.00 & \\
\hline \multirow[t]{2}{*}{ Age group } & $15-24$ years & $11(91.7)$ & $8(42.1)$ & $14.24[4.02-50.38]$ & $<0.001$ & $12.35[3.90-39.11]$ & $<0.001$ \\
\hline & $25-49$ years & $1(8.3)$ & $11(57.9)$ & 1.00 & & 1.00 & \\
\hline
\end{tabular}

Transmission patterns could not be included because it caused the model not to converge ( 12 cases, 110 controls, 5 communes)

Deployment of LLIN and IRS may have prevented 100,000 cases annually. Note that IRS in Madagascar is mainly deployed in low-transmission settings and LLIN in almost all the country, which explains why the number of prevented cases differs so much between the two MCI while PE are similar. Even though it may be regarded as simplistic to add cases avoided by LLIN to cases avoided by IRS, these results give a rough overview of the number of prevented cases, and they could feed more sophisticated cost-effectiveness or transmission models.

The present results are close to results from a simultaneous CSS survey aimed at estimating the effectiveness of MCI against Plasmodium infection where LLIN was found to have a $41 \% \mathrm{PE}$, IRS had a $44 \% \mathrm{PE}$ at household level in certain places, the concurrent exposure to both LLIN and IRS had a $86 \%$ PE, and IPTp a $65 \%$ PE $[14,15]$. This suggests that measuring the effectiveness of MCI for one outcome, e.g. malaria infection is a reasonable proxy for the other, e.g. clinical malaria. As observed in the study mentioned above, the southern part of Madagascar appears to be different from the other zones, but the present study lacked statistical power to evaluate the effectiveness of LLIN in this transmission pattern since the sample size of cases was eight only.

The results from the present study are also in line with results from efficacy studies of LLIN and IRS, which suggests that the efficacy of MCI is preserved in Madagascar. In meta-analyses, insecticides-treated nets (ITN) were shown to provide a $50 \% \mathrm{PE}$ against malaria incidence as compared to no nets in areas of stable malaria, and a $62 \%$ PE in areas of unstable malaria [20], and the PE of IRS ranged between 31 and $88 \%$ in areas of unstable malaria [21]. Nevertheless, in contrast with these studies, the present results show no relationship between transmission patterns and MCI effectiveness. This might be explained by an insufficient statistical power in the present study to detect such a difference, since few cases have been identified in low-transmission areas. In the present study, the 
Table 5 Estimation of the annual number of clinical malaria cases prevented by vector control interventions in Madagascar

\begin{tabular}{|c|c|c|c|c|c|c|c|c|c|c|}
\hline \multirow{2}{*}{$\begin{array}{l}\text { Transmission } \\
\text { pattern }\end{array}$} & \multirow{2}{*}{$\begin{array}{l}\mathrm{N} \text { cases } \\
\text { in } 2011\end{array}$} & \multicolumn{4}{|l|}{ LLIN } & \multicolumn{4}{|l|}{ IRS } & \multirow{2}{*}{$\begin{array}{l}\text { Total } \mathrm{N} \text { cases } \\
\text { prevented }\end{array}$} \\
\hline & & $\mathrm{PE}(\%)$ & Cov. (\%) & CE (\%) & $\begin{array}{l}\mathrm{N} \text { cases pre- } \\
\text { vented }\end{array}$ & PE (\%) & Cov. (\%) & CE (\%) & $\begin{array}{l}\mathrm{N} \text { cases pre- } \\
\text { vented }\end{array}$ & \\
\hline East & 157,858 & 51 & 61.1 & 31 & 71,457 & NA & 3.8 & NA & NA & 71,457 \\
\hline Fringe & 8347 & 51 & 35.4 & 18 & 1839 & 51 & 58.6 & 30 & 3558 & 5397 \\
\hline West & 40,068 & 51 & 55.7 & 28 & 15,898 & 51 & 18.3 & 9 & 4124 & 20,023 \\
\hline South & 8481 & -30 & 41.9 & NA & NA & 51 & 67.8 & 35 & 4483 & 4483 \\
\hline Highlands & 6297 & NA & 7.7 & NA & NA & 51 & 43.1 & 22 & 1774 & 1774 \\
\hline Total & 221,051 & & & & 89,194 & & & & 12,165 & 101,359 \\
\hline
\end{tabular}

$P E$ protective effectiveness, CE community effectiveness, NA not applicable

age under 5 years was not associated with a better effectiveness of MCI, while this is theoretically expected [22] and observed in some efficacy studies [23].

This study design was suggested more than a decade ago in order to monitor the effectiveness of MCI through time and space $[8,9]$, but relatively few surveys have been conducted. The scarceness of CCS is surprising given the relative simplicity of the survey design and importance of the information it provides in terms of policy guidance. If vector control interventions are consider, for example, only 13 CCS have tried to measure the PE of ITN [9, 2434 , and four the PE of IRS-including two of the previous [24, 25, 35, 36]. Only six of these 15 surveys collected data in a geographical region of the size of a district or above, and their sample sizes were smaller (mean number of cases 219, range 35-534), which makes the present study the biggest CCS ever aimed at evaluating the effectiveness of $\mathrm{MCI}$, with a unique countrywide design.

Nine of the CCS mentioned above found a significant PE of ITN. As compared with these studies, the use of bed net was defined more restrictively, i.e. the use every night in the last 3 months instead of the use last night. This definition was selected in order to provide a more accurate estimation of the effectiveness in the perspective of being used for mathematical modelling or for calculation of the cost-effectiveness ratio. Indeed, the "last night" definition is more sensitive to social desirability bias and clerical errors, and tends to overestimate the coverage [10].

This study presents several limitations. One of the main issues is that a minimal questionnaire was used in order to facilitate the practitioners' task, preventing us from collecting useful data such as socioeconomic status or education level for adjustment of multivariate models. Nevertheless, in a concurrent study aimed at evaluating the effectiveness of the very same MCI against infection through a CSS design, the difference between crude and adjusted OR were trivial.
The results of the study might have underestimated the actual effectiveness of LLIN and IRS for four reasons. Firstly, the study design does not allow for calculation of herd protection offered by vector control interventions while some authors claim that the protection offered by these interventions is more visible at the community than at the individual or household level, especially IRS [37]. Secondly, the survey did not cover the period during which IRS is expected to have an effect on vectors, i.e. a few months after spraying [38]. The previous IRS campaigns took place in November-December 2011, and one happened at the end of the period of data collection, in March-April 2013. The latter campaign was supposed to take place in November-December 2012, but was delayed. The low coverage (i.e. household sprayed last 12 months) of cases in summer might thus reflect a long interval since the previous IRS campaign, and the PE derived from these cases is probably not reliable. The most comparable cases with controls are definitely those who have been identified in the spring of 2012, i.e. at the same moment as the CSS. When the analysis was restrained to these individuals, the PE of IRS was demonstrated. As other authors have shown, the duration of the bio-efficacy of IRS was not expected to last more than 6-8 months [38], but its impact on malaria incidence may remain after the disappearance of the insecticide effect $[14,39,40]$. Third, LLIN use in controls was estimated in a season where bed net use was low $[10,41]$. Nevertheless, non-significant interaction terms indicate that season did not influence the measure of the effectiveness of LLIN. Fourth, a recall bias might have occurred, as for all case-control studies. If cases better recalled exposure to MCIs, or if memory failures occurred in both cases and controls, then hypothesis tests would be biased towards the null [42].

Another limitation is the selection of cases and controls. In a theoretical database of over 6000 malaria cases, only 841 were retained, mainly because 
practitioners who agreed to participate in the study did not systematically fill in questionnaires. Nevertheless, since the choice of submitting the questionnaire to the patient or not was likely not driven by the exposure to MCI or confounding factors related to the place, one can reasonably suppose that no systematic bias occurred. Regarding controls, they are not strictly comparable to cases since they have not been selected in the operational catchment population of $\mathrm{SHC}$, i.e. the individuals who get to the SHC when they are affected by febrile illnesses, but in the geographical catchment population, i.e. living in the same commune as cases. Typically, coverage of the three MCI investigated here were higher in less populated areas [10] who have a likely lower access to health facilities; this might have resulted in an overestimation of the effectiveness of MCI. Recruiting controls among non-malaria patients or persons accompanying patients seen in the same health structures as patients would avoid this bias.

Finally, the present study has serious limitations in its statistical power regarding IPTp since the sample sizes are small. Therefore, the non-significant OR of IPTp shall not be considered as reflecting a poor effectiveness but rather as the reflection of an insufficient statistical power. Moreover the short questionnaire did not allow for the collection of data about parity, which would have been preferable to adjust for. Similarly, the non-significant additional effect of both LLIN and IRS over one single intervention might be attributed to a lack of statistical power since the analysis has been conducted on a small subset of the whole database.

Despite these limitations, the strengths of this studyits large sample size, its countrywide scale, and its population-wide representativeness-suggest that the results are valuable for policy guidance in Madagascar. They allow, for the first time, an estimation of the number of clinical cases that have avoided by preventive measures. That opens the way for cost-effectiveness analysis that could help the health authorities in the choice of funding the MCI.

\section{Conclusions}

The results of this study showed that, in Madagascar in 2012, both LLIN and IRS provided good protective effectiveness against clinical malaria. The study failed to prove that combining both MCI simultaneously might provide additional effect. However, combining these two vector control interventions might be indicated if the coverage or the effectiveness of one of them is problematic. The present study also could prompt stakeholders to fund operational research in other endemic countries, in order to verify the local performance of MCI by case-control approaches.

\section{Additional file}

Additional file 1. Effectiveness of LLIN in all areas with LLIN distribution. $\mathrm{Bi}$ - and multivariate analyses of risk factors for developing a clinical malaria episode, including bed net use.

\section{Abbreviations}

CCS: case-control study; CE: community effectiveness; CSS: cross-sectional survey; GEE: generalized estimating equations; IPTp: intermittent preventive treatment in pregnancy; ITN: insecticide-treated nets; IRS: indoor residual spraying; LLIN: long-lasting insecticidal nets; MCI: malaria control interventions; MEDALI: Mission d'Etude des Déterminants de l'Accès aux Méthodes de Lutte antipaludique et de leur Impact; NIBN: non-impregnated bed net; OR: odds ratio; PE: protective effectiveness; RCT: randomized controlled trial; RDT: rapid diagnostic tests; SHC: sentinel health centres.

\section{Authors' contributions}

CR and TK designed the CSS questionnaire, TK, MR and VR conducted the CSS, and TK analyzed the data from the CSS. CR, TK, and LR designed the CCS questionnaire, TK, LR, PP, and MR conducted the CCS, and TK and VR analyzed the data from the CCS. CR designed the MEDALI project and the present analysis. TK coordinated the MEDALI project overall and drafted the manuscript. All authors conceived the study and contributed to the interpretation of data. All authors read and approved the final manuscript.

\section{Author details}

${ }^{1}$ Malaria Research Unit, Institut Pasteur de Madagascar, BP 1274, 101 Avaradoha, Antananarivo, Madagascar. ${ }^{2}$ Unité de recherche sur les maladies infectieuses et tropicales émergentes (URMITE) - UMR 6236, 27 boulevard Jean Moulin, 13385 Marseille Cedex 05, France. ${ }^{3}$ Fondation Mérieux, 17 rue Bourgelat, 69002 Lyon, France. ${ }^{4}$ Epidemiology Unit, Institut Pasteur de Madagascar, BP 1274, 101 Avaradoha, Antananarivo, Madagascar. ${ }^{5}$ Institute for Biomedical Research of the French Armed Forces (IRBA), BP 73, 91223 Brétigny-Sur-Orge Cedex, France.

\section{Acknowledgements}

This research was supported by the Global Fund to Fight AIDS, Tuberculosis and Malaria via the Initiative $5 \%$ programme initiated by the French Ministry of Foreign Affairs and France Expertise Internationale (Grant \#12INI109). We especially thank the population of Madagascar who participated the study. We thank those who facilitated the survey, i.e. the fokonolona, heads of fokontany, local administration authorities and health authorities from Ministry of Health and National Malaria Control Programme (NMCP). We must also thank the NMCP for providing incidence figures. Our socio-anthropology team (C. Mattern, E. Raboanary, and D. Pourette) deserve special thank for their help in the design of the questionnaire and useful discussion about results. We thank also the survey teams and members of the Monitoring Committee, i.e. P. Tafangy, B. Ramarosandratana, P. Rajaonarison, A. Finlay, L. P. Rabarijaona, S. Zigirumugabe, A. Ratsimbasoa, R. Andrianasolo, A. Lamina, J. D. Rahariniaina, S. A. Rafalimanantsoa, E.E. Rambolamanana, M. C. Rakotoarivony, H. Razafindranaivo, M. Rabary, S. Goedefroit, T. Andrianatoandro, N. Elissa, J. Rakotonjato, J. A. Ravelonarivo, F. Rakotomanana, P. L. Lesage, F. Sandron, M. Gaimard, C. Bellas-Cabane, and B. Meslet.

\section{Competing interests}

The authors declare that they have no competing interests.

Received: 7 July 2015 Accepted: 27 January 2016

Published online: 11 February 2016

\section{References}

1. WHO Global Malaria Programme. World malaria report 2014. Geneva: World Health Organization; 2014.

2. Ministère de la Santé Publique, Programme National de Lutte contre le Paludisme. Revue du programme paludisme à Madagascar. Antananarivo; 2011. 
3. Steketee RW, Campbell CC. Impact of national malaria control scale-up programmes in Africa: magnitude and attribution of effects. Malar J. 2010;9:299.

4. Hay SI, Guerra CA, Tatem AJ, Atkinson PM, Snow RW. Urbanization, malaria transmission and disease burden in Africa. Nat Rev Microbiol. 2005;3:81-90.

5. Caminade C, Kovats S, Rocklov J, Tompkins AM, Morse AP, Colón-González FJ, et al. Impact of climate change on global malaria distribution. Proc Natl Acad Sci USA. 2014;111:3286-91.

6. Tusting LS, Willey B, Lucas H, Thompson J, Kafy HT, Smith R, et al. Socioeconomic development as an intervention against malaria: a systematic review and meta-analysis. Lancet. 2013;382:963-72.

7. D'Acremont V, Kahama-Maro J, Swai N, Mtasiwa D, Genton B, Lengeler C. Reduction of anti-malarial consumption after rapid diagnostic tests implementation in Dar es Salaam: a before-after and cluster randomized controlled study. Malar J. 2011;10:107.

8. Lengeler C, Snow R. From efficacy to effectiveness: insecticide-treated bednets in Africa. Bull World Health Organ. 1996;74:325-32.

9. Rogier C, Henry M-C, Luxemburger C. Méthodes d'évaluation en phase IV de l'efficacité de la lutte antivectorielle: l'exemple d'une étude cas-témoin de l'impact des moustiquaires imprégnées d'insecticide à longue durée d'action après leur déploiement au Bénin. Med Trop (Mars). 2009;69:195-202.

10. Kesteman T, Randrianarivelojosia M, Mattern C, Raboanary E, Pourette D, Girond F, et al. Nationwide evaluation of malaria infections, morbidity, mortality, and coverage of malaria control interventions in Madagascar. Malar J. 2014;13:465.

11. Institut National de la Statistique, Programme National de Lutte contre le Paludisme, ICF International. Enquête sur les indicateurs du paludisme à Madagascar 2011. Antananarivo; 2012.

12. Randrianasolo $L$, Raoelina $Y$, Ratsitorahina $M$, Ravolomanana $L$, Andriamandimby S, Heraud J-M, et al. Sentinel surveillance system for early outbreak detection in Madagascar. BMC Public Health. 2010;10:31.

13. Oyakhirome S, Profanter K, Kremsner PG. Assessment of fever in African children: implication for malaria trials. Am J Trop Med Hyg. 2010;82:215-8.

14. Kesteman T, Randrianarivelojosia M, Piola P, Rogier C. Post-deployment effectiveness of malaria control interventions on Plasmodium infections in Madagascar: a comprehensive phase IV assessment. (In preparation).

15. Kesteman T, Randrianarivelojosia M, Piola P, Rogier C. Assessing the impact of vector control interventions by measuring their effectiveness - what has been done in Madagascar. Malar J. 2014;13(Suppl 1):05.

16. Harrell FEJ: bpower: power and sample size for two-sample binomial test. In: Package Hmisc. 2014. p. 28-30. http://CRAN.R-project.org/ package $=$ Hmisc.

17. Carey VJ, Lumley T, Ripley B. gee: generalized estimation equation solver. $R$ package version 4.13-18. 2012

18. Gelman A, Goegebeur Y, Tuerlinckx F, Van Mechelen I. Diagnostic checks for discrete data regression models using posterior predictive simulations. Appl Stat. 2000;49:247-68.

19. Gelman A, Su Y-S. arm: data analysis using regression and multilevel/hierarchical models. R package version 1.6-09. 2013.

20. Lengeler $C$. Insecticide-treated bed nets and curtains for preventing malaria. Cochrane Database Syst Rev. 2004;2:CD000363.

21. Pluess B, Tanser FC, Lengeler C, Sharp BL. Indoor residual spraying for preventing malaria. Cochrane Database Syst Rev. 2010:4:CD006657.

22. Snow RW, Marsh K. The consequences of reducing transmission of Plasmodium falciparum in Africa. Adv Parasitol. 2002;52:235-64.

23. Curtis C, Maxwell C. A comparison of use of a pyrethroid either for house spraying or for bednet treatment against malaria vectors. Trop Med Int Health. 1998;3:619-31.

24. Macedo De Oliveira A, Mutemba R, Morgan J, Streat E, Roberts J, et al. Prevalence of malaria among patients attending public health facilities in Maputo City, Mozambique. Am J Trop Med Hyg. 2011;85:1002-7.
25. Yamamoto SS, Louis VR, Sié A, Sauerborn R. The effects of zooprophylaxis and other mosquito control measures against malaria in Nouna, Burkina Faso. Malar J. 2009;8:283.

26. Abdella YM, Deribew A, Kassahun W. Does insecticide treated mosquito nets (ITNs) prevent clinical malaria in children aged between 6 and 59 months under program setting? J Community Health. 2009;34:102-12.

27. Mathanga DP, Campbell CH, Taylor TE, Barlow R, Wilson ML. Reduction of childhood malaria by social marketing of insecticide-treated nets: a case-control study of effectiveness in Malawi. Am J Trop Med Hyg. 2005;73:622-5.

28. Alexander N, Rodriguez M, Pérez L, Caicedo JC, Cruz J, Prieto G, et al. Case-control study of mosquito nets against malaria in the Amazon region of Colombia. Am J Trop Med Hyg. 2005;73:140-8.

29. Rowland M, Webster J, Saleh P, Chandramohan D, Freeman T, Pearcy B, et al. Prevention of malaria in Afghanistan through social marketing of insecticide-treated nets: evaluation of coverage and effectiveness by cross-sectional surveys and passive surveillance. Trop Med Int Health. 2002;7:813-22.

30. Armstrong Schellenberg JRM, Abdulla S, Nathan R, Mukasa O, Marchant TJ, Kikumbih N, et al. Effect of large-scale social marketing of insecticide treated nets on child survival in rural Tanzania. Lancet. 2001;357:1241-7.

31. Rowland M, Hewitt S, Durrani N, Saleh P, Bouma M, Sondorp E. Sustainability of pyrethroid-impregnated bednets for malaria control in Afghan communities. Bull World Health Organ. 1997;75:23-9.

32. Oladeinde BH, Omoregie R, Olley M, Anunibe JA, Onifade AA. Malaria and anemia among children in a low resource setting in Nigeria. Iran J Parasitol. 2012;7:31-7.

33. Okebe J, Mwesigwa J, Kama EL, Ceesay SJ, Njie F, Correa S, et al. A comparative case control study of the determinants of clinical malaria in The Gambia. Malar J. 2014;13:306.

34. Abdulla S, Schellenberg JRMA, Mukasa O, Lengeler C. Usefulness of a dispensary-based case-control study for assessing morbidity impact of a treated net programme. Int J Epidemiol. 2002;31:175-80.

35. Guthmann J-P, Hall AJ, Jaffar S, Palacios A, Lines J, Llanos-Cuentas A. Environmental risk factors for clinical malaria: a case-control study in the Grau region of Peru. Trans R Soc Trop Med Hyg. 2001;95:577-83.

36. Sharma PK, Ramanchandran R, Hutin YJ, Sharma R, Gupte MD. A malaria outbreak in Naxalbari, Darjeeling district, West Bengal, India, 2005: weaknesses in disease control, important risk factors. Malar J. 2009;8:288.

37. Rehman AM, Coleman M, Schwabe C, Baltazar G, Matias A, Gomes IR, et al. How much does malaria vector control quality matter: the epidemiological impact of holed nets and inadequate indoor residual spraying. PLoS One. 2011;6:e19205.

38. Okumu FO, Chipwaza B, Madumla EP, Mbeyela E, Lingamba G, Moore J, et al. Implications of bio-efficacy and persistence of insecticides when indoor residual spraying and long-lasting insecticide nets are combined for malaria prevention. Malar J. 2012;11:378.

39. Ratovonjato J, Randrianarivelojosia M, Rakotondrainibe ME, Raharimanga V, Andrianaivolambo L, Le Goff G, et al. Entomological and parasitological impacts of indoor residual spraying with DDT, alphacypermethrin and deltamethrin in the western foothill area of Madagascar. Malar J. 2014;13:21.

40. Bukirwa H, Yau V, Kigozi R, Filler S, Quick L, Lugemwa M, et al. Assessing the impact of indoor residual spraying on malaria morbidity using a sentinel site surveillance system in Western Uganda. Am J Trop Med Hyg. 2009;81:611-4.

41. Mattern C, Raboanary E, Kesteman T, Rogier C, Piola P, Pourette D. Malaria is not a problem. Consequences on fever case management and use of bed nets in Madagascar. PLoS One. 2016. Manuscript accepted.

42. Raphael K. Recall bias: a proposal for assessment and control. Int J Epidemiol. 1987;16:167-70. 